\title{
In Situ Preparation and Immobilization of Semiconducting Polymer Dots on Microbeads for Efficient and Stable Photocatalytic Hydrogen Evolution
}

\author{
Aijie Liu, Lei Tian, and Haining Tian* \\ Cite This: ACS Appl. Energy Mater. 2021, 4, 4308-4312 \\ Read Online
}

ACCESS | Lلll Metrics \& More | 国 Article Recommendations ｜ sl Supporting Information

ABSTRACT: Organic semiconducting polymers dots (Pdots) have recently shown efficient photocatalytic activity for hydrogen evolution in an aqueous phase. However, colloidal Pdots face problems of aggregation and precipitation during the photocatalytic reaction due to unavoidable collisions between particles, thus resulting in a short catalytic lifetime. In this work, in situ preparation of PFBT Pdots on the surface of microbeads is reported. Results indicate that, with this facile method and support of a template, the photocatalytic properties of PFBT Pdots can be highly enhanced.

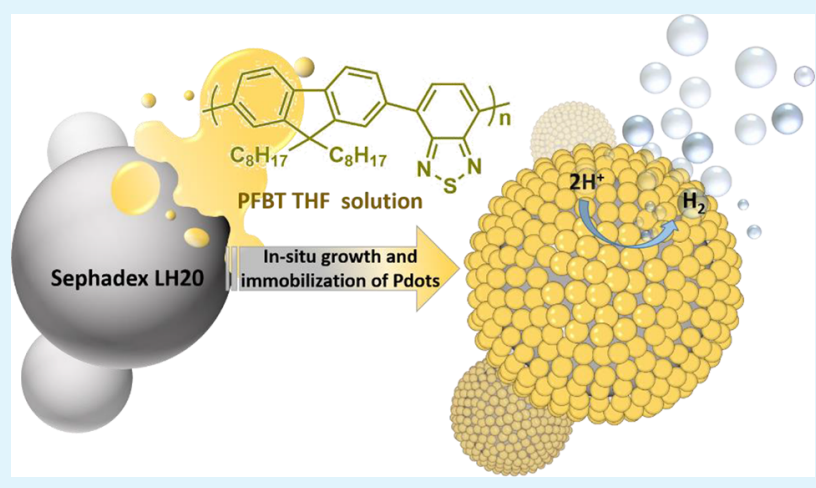

KEYWORDS: surface immobilization, Pdots, microbeads, photocatalysis, hydrogen evolution

$\mathrm{W}$ ith the increasing global energy demand, clean and renewable solar energy has shown great potential as a promising alternative energy source to fossil fuels in order to meet the worldwide energy demand and sustainable development. In nature, photosynthesis systems convert solar energy into storable chemical energy by converting $\mathrm{CO}_{2}$ and water from the wild environment into carbohydrate fuels and oxygen with a high efficiency. ${ }^{1,2}$ Inspired by nature, artificial photocatalysts have been developed to harvest sunlight efficiently and transform it into chemical fuels. ${ }^{3}$ Among nanophotosynthetic systems, nanometal oxides, ${ }^{4}$ metal nanoparticles, ${ }^{5}$ quantum dots, and their composite nanomaterials have attracted great attention due to their large surface area that provides an enormous amount of surface active sites. ${ }^{6}$ Semiconducting polymers have been used in photocatalysis for water splitting, ${ }^{9} \mathrm{CO}_{2}$ reduction, ${ }^{8,9}$ photoredox reactions, ${ }^{10}$ as well as water treatment. ${ }^{11}$ Making the semiconducting polymer into small particles is a conventional way to increase the photocatalytic activity due to an enhanced active surface area and short exciton diffusion path. ${ }^{12,13}$ Since water is considered the greenest solvent for photocatalysis due to its nontoxicity and role as a proton resource, a hydrophilic surface and excellent dispersibility of the polymer particles are therefore vital to enhance photocatalytic reactions in water. ${ }^{14,15}$ Adding water-miscible organic solvents to water is a traditional way to increase the dispersibility of the pristine hydrophobic polymers that cannot be dissolved or dispersed in water for photocatalysis. However, the addition of organic solvent is not a green method for the development of sustainable energy.
Recently, assembling a polymer into small hydrophilic nanodots (Pdots) by use of a so-called nanoprecipitation method has been proven to be an effective strategy to enhance the photocatalysis, ${ }^{16-21}$ particularly for light-driven hydrogen evolution. The formed hydrophilic polymer particles have several advantages to enhance the photocatalysis: (1) Polymers self-assembled into small particles are able to increase active sites which benefit photocatalysis such as proton reduction having a proton-proton coupling reaction. (2) Use of an amphiphilic polymer creates proton channels in the particles that allow a free proton to diffuse and interact with active sites. $^{22}$ (3) Porous nanoparticles can significantly shorten the exciton diffusion path. However, there are also drawbacks for the nanoprecipitation method: (1) Plenty of organic solvent is needed due to the narrow Ouzo region. (2) It is hard to obtain a concentrated polymer particle solution in a one-step preparation due to particle size change and even serious particle aggregation and precipitation from a highly concentrated solution. (3) Reactivity is lost rapidly from unwanted aggregation and precipitation forming big particles during photocatalysis. In order to overcome these drawbacks

Received: March 5, 2021

Accepted: April 22, 2021

Published: April 27, 2021 


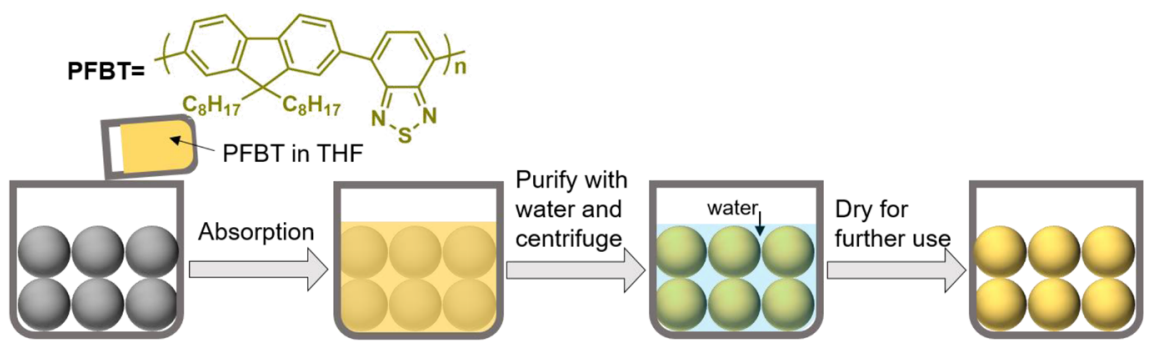

Figure 1. Scheme of in situ preparation of PFBT Pdots with Sephadex LH-20 microbeads as templates.

mentioned above, herein we report a facile method to enhance the photocatalytic stability of the PFBT (also called F8BT) semiconducting polymers by in situ preparation and immobilization of Pdots on the microbead templates (see Figure 1).

In this work, commercially available cross-linked dextran beads, Sephadex LH-20 microbeads were used as a platform for the in situ preparation and immobilization of PFBT Pdots. Sephadex LH-20 is designed for molecular sizing of natural products and is low-cost and environmentally friendly. Owing to the nature of both hydrophilic and lipophilic characters, the large water absorption ability makes it a good candidate for conducting polymer immobilization as well as for the application of hydrogen evolution from water.

The preparation of Pdot immobilized hybrid microbeads involves several simple steps: First, $200 \mu \mathrm{L}$ of $1 \mathrm{mg} / \mathrm{mL}$ PFBT THF solution was added into $100 \mathrm{mg}$ of Sephadex microbeads; conjugated polymers absorbed on the surface of microbeads are attributed to the lipophilic nature of the matrix, and the large molecular size of PFBT is able to prevent them from entering mesopores of the microbeads. Second, a large excess of water was added quickly; the follow-up purification step was carried out by using a centrifuge to remove solvents as well as the unabsorbed polymers. Finally, the PFBT coated hybrid microbeads were slowly dried under $35{ }^{\circ} \mathrm{C}$ and stored under room temperature for further use. The loading efficiency of the polymer on the microbeads is around $85 \%$ (see SI for loading calculation details), resulting in PFBT loading of $0.17 \mathrm{wt} \%$. Note that, due to the fact that the stock solution of PFBT in THF used to prepare the hybrid-microbeads has an optimal concentration of $1 \mathrm{mg} / \mathrm{mL}$, and the highest volume of THF that can be absorbed by $100 \mathrm{mg}$ of microbeads is $200 \mu \mathrm{L}$ at ambient conditions, the highest loading of PFBT is determined to be about $170 \mu \mathrm{g}$ per $100 \mathrm{mg}$ of microbeads in this work, while particles with lower loadings were also obtained by mixing $200 \mu \mathrm{L}$ of $n(n<1) \mathrm{mg} / \mathrm{mL}$ PFBT THF solution with $100 \mathrm{mg}$ of Sephadex microbeads. Results and discussions presented in the following context are with the highest loading, unless otherwise noted.

The purity of hybrid microbeads was confirmed by using a fluorescence microscope to give a visualized perspective. The fluorescence microscope images were taken under the excitation wavelength of $458 \mathrm{~nm}$ and detection wavelength between 500 and $700 \mathrm{~nm}$. Fluorescent hybrid microbeads were highlighted in contrast to the clean and dark background (Figure 2a); consistent with the white image (Figure $2 \mathrm{~b}$ ), a clean background was observed. Results indicate that all nonattached polymers were successfully removed in the purification step. The inserted image in Figure 2a with a higher magnification shows relatively uniform fluorescence

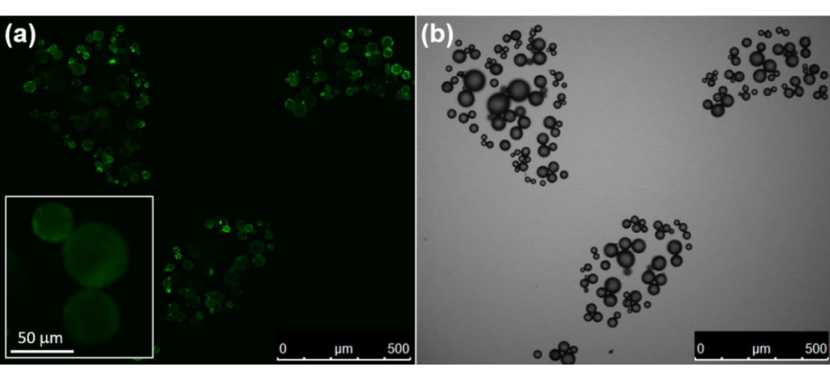

Figure 2. Fluorescence microscope images of hybrid microbeads with excitation wavelength $458 \mathrm{~nm}$ laser, and emission was detected between 500 and $700 \mathrm{~nm}$ (a). White image (b).

locating over all of the microbeads, indicating a homogeneous distribution of polymers around the microbeads.

The morphology of hybrid microbeads was analyzed by a scanning electron microscope (SEM). Sephadex LH-20 microbeads show a hickory nut-like structure with a smooth surface (Figure 3a). After a coating of PFBT polymer, PFBT Pdots were formed and decorated at the surface of the microbeads, as shown in Figure $3 b, c$, while in general, a smooth polymer shell is more commonly observed by using in situ polymerization of a conducting polymer on a microbead template. ${ }^{23}$ More importantly, with this in situ preparation and immobilization method, highly monodisperse Pdots with an average size of $70 \mathrm{~nm}$ were obtained (from the Gaussian fit in Figure 3d). Note that Pdot formation with a small size is rather important for photocatalytic reactions, and the size dependent photocatalytic activity of Pdots has been reported by our group as well as others. ${ }^{24,25}$ In contrast, unwanted precipitation was observed by using a general nanoprecipitation method under a high concentration of $1 \mathrm{mg} / \mathrm{mL}$ PFBT (data not shown), and Pdots with large sizes were formed when the polymer concentration is above $100 \mu \mathrm{g} / \mathrm{mL}$ (Figure S1). The hybrid microstructure was maintained after several centrifuge steps, which also indicates a strong interaction between the PFBT Pdots and microbeads, resulting in highly stable Pdot coated hybrid microbeads. Under the loading of $170 \mu \mathrm{g}$ of PFBT per $100 \mathrm{mg}$ of microbeads, random assembling of Pdots and partial coating were also observed (shown in Figure S2a). Individual Pdots homogeneously dispersed on the surface of microbeads were observed under low loading (Figure S2b).

The possible pathway for Pdot formation and their selfassembly around microbeads is described as follows. First, nanosized Pdots were formed right after adding water (in the purification step); this step is similar to a traditional nanoprecipitation method. ${ }^{26}$ The majority of the organic solvent was absorbed by microbeads and left polymers absorbed on the lipophilic surface. Adding water allows a rapid mix, forming uniform polymer particles, which usually 


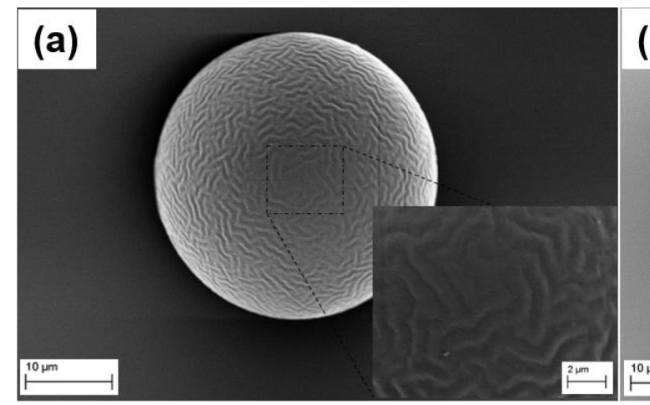

(b)

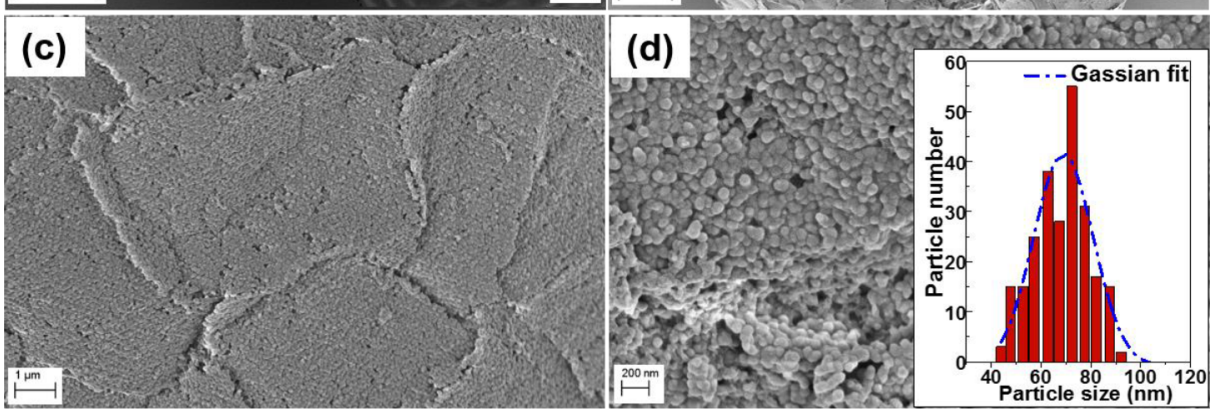

Figure 3. SEM images. (a) Sephadex LH-20 and $(b-d)$ in situ immobilized Pdots on Sephadex LH-20 with an increasing magnification. Particle size distribution (inset) was obtained by counting 200 particles from the image (d).

can be achieved by using a microfluidic rapid mixing method. ${ }^{27}$ Both the surface environment and lipophilic properties of cross-linked dextran may contribute to the formation of Pdots under a high polymer concentration. It is difficult for the traditional nanoprecipitation method; since concentration usually plays a key role in the determination of particle size, and a uniform growth of particles can only proceed within the boundaries of the narrow Ouzo region, unwanted aggregations causing precipitation of Pdots from water were often observed at high supersaturation. ${ }^{28}$ Second, self-assembly of hydrophobic nanoparticles into spherical clusters around microbeads may be similar to the Ostwald ripening process; ${ }^{29}$ the selfassembly is induced by removal of the THF organic phase and slow evaporation of water during the purification and drying step. ${ }^{30}$ Again, the lipophilic nature of cross-linked dextran probably offers proper interactions between Pdots, allowing self-assembly and immobilization. This method offers precise control over the conducting polymer PFBT loading and gives a uniform coating to a certain degree, although the amount of loading is lower than in situ polymerization of the conducting polymer layer; ${ }^{31}$ however, polymer nanoparticles and their assemblies that are presented in this work give a much higher surface area as compared to smooth polymer layers. A larger catalytic surface area and short exciton diffusion path were suggested to benefit photocatalytic activity. ${ }^{22}$

The absorption and fluorescence emission spectra of Pdot assemblies on microbeads were measured, as shown in Figure S3. Absorption broadening was found for Pdot assemblies under the highest loading as well as lower loadings (Figure S4). It may indicate that this broadening absorption is not due to particle clustering but is from the aggregation of PFBT into a single particle. There is only a small red-shift in fluorescence emission for Pdot assemblies compared to free PFBT in THF (Figure S3). A time-correlated single photon counting (TCSPC) study shows a similar excitation lifetime for PFBT Pdot assemblies under a wide range of particle loading (Figure S5), which indicates that Pdot assemblies in this work do not result in more exciton annihilation from the aggregated morphology. This should benefit the photocatalytic reaction under high Pdot loading.

Hydrogen evolution of hybrid microbeads with $170 \mu \mathrm{g}$ loading was analyzed by gas chromatography (GC), as shown in Figure 4. The hydrogen evolution with lower PFBT loading was recorded by a Clark-type hydrogen sensor instead, due to the low amount of hydrogen generation, as shown in Figure S6. With the stabilization of microbeads, the Pdots with $170 \mu \mathrm{g}$ loading remained active for at least $80 \mathrm{~h}$, resulting in a much higher amount of hydrogen production (Figure 4, blue circle). The average photocatalytic reactivity for the first $20 \mathrm{~h}$ is about

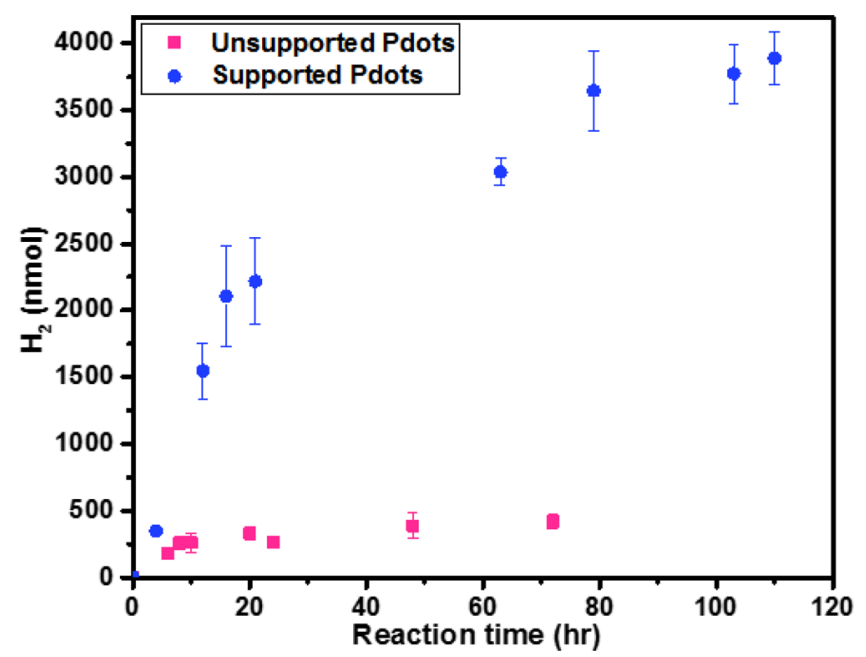

Figure 4. Visible-light-driven hydrogen generation from water at room temperature. Conditions: $170 \mu \mathrm{g}$ of PFBT Pdots was loaded on $100 \mathrm{mg}$ of Sephadex LH20; ascorbic acid, 0.6 M; pH 4; $3 \mathrm{~mL}$ in a 9 $\mathrm{mL}$ airtight vial; LED lamp, white light $\left(50 \mathrm{~mW} \mathrm{~cm}^{-2}, 420-750 \mathrm{~nm}\right)$; blue circle, supported Pdots, hybrid microbeads; purple square, unsupported free Pdots (170 $\mu \mathrm{g}$ of PFBT Pdots; ascorbic acid, $0.6 \mathrm{M}$; $\mathrm{pH} 4 ; 3 \mathrm{~mL}$ in $9 \mathrm{~mL}$ airtight vial). $\mathrm{H}_{2}$ generation collected with gas chromatography (GC) analysis. Three independent measurements were taken at each data point. 
$700 \mu \mathrm{mol} /(\mathrm{g} \mathrm{h})$; a further decrease in the activity may be due to the degradation of PFBT. No detectable amount of hydrogen was found for bare LH20 microbeads, which indicates that the surface immobilized PFBT Pdots are responsible for the hydrogen evolution, while free PFBT Pdots tend to aggregate (Figure S7) under the photocatalytic condition and lose catalytic activity within a few hours (Figure 4, purple square). The photocatalytic activity of pristine polymer in water was also carried out. However, due to the poor solubility (or dispersity) of the pristine polymer in the aqueous phase, no detectable amount of hydrogen was observed, which is consistent with the previous result that very little activity (from a hydrogen sensor) was found for pristine polymer. ${ }^{13}$ Nanoparticles with a large surface area and short exciton diffusion path therefore play a key role for the enhancement of activity, and a stable hybrid microsystem presented in this work contributes to the significantly enhanced catalytic lifetime. Moreover, the hydrogel property of the LH20 microbead provides a hydrophilic support which may also be important for PFBT Pdots interacting with protons for hydrogen evolution. This results in a reasonable external quantum yield (EQY) of $0.43 \%$ at $420 \mathrm{~nm}$ and $0.24 \%$ at $450 \mathrm{~nm}$ (Figure $\mathrm{S} 8$ ). Water contact angle analysis shows that LH20 microbeads are highly hydrophilic and absorb water easily; no detectable water contact angle was found, and the water droplet was absorbed by LH20 immediately (Figure S9). After coating with PFBT, the surface hydrophilicity decreased, giving an initial contact angle of $98^{\circ}$. However, due to the high water absorbability of $\mathrm{LH} 20$, the water contact angle decreased over time quickly, down to $17^{\circ}$ at $1.3 \mathrm{~s}$, and the water droplet was completely absorbed by LH20 in 3.4 s, as shown in Figure S10.

In summary, we have successfully prepared hybrid microbeads by using the in situ growth of PFBT Pdots on the surface of commercially available and low-cost cross-linked dextran beads, Sephadex LH-20 microbeads. Owing to the nature of both hydrophilic and lipophilic characters, a semiconducting polymer PFBT was adsorbed on the surface of microbeads; Pdots and their clusters were formed along the microbeads. With the assistance of the microbead template, the immobilized Pdots increase the hydrogen production as compared to the colloidal Pdots. The active time has been elongated from several hours to more than $80 \mathrm{~h}$. This method also provides a strategy to combine photocatalysts for water oxidation and proton/ $\mathrm{CO}_{2}$ reduction on the conducting nanoparticle surface for full water splitting or $\mathrm{CO}_{2}$ reduction using water as a proton and electron source in the future.

\section{ASSOCIATED CONTENT}

\section{SI Supporting Information}

The Supporting Information is available free of charge at https://pubs.acs.org/doi/10.1021/acsaem.1c00650.

Preparation of Pdots in aqueous and on microbeads, photocatalytic experiment, fluorescence microscopy, steady-state absorption and fluorescence measurements, dynamic light scattering measurements, TCSPC, SEM measurements, EQY measurement, and contact angle measurements (PDF)

\section{AUTHOR INFORMATION}

\section{Corresponding Author}

Haining Tian - Department of Chemistry-Ångström Laboratory, Uppsala University, SE 75120 Uppsala, Sweden; (ㅇ orcid.org/0000-0001-6897-2808;

Email: haining.tian@kemi.uu.se

\section{Authors}

Aijie Liu - Department of Chemistry-Ångström Laboratory, Uppsala University, SE 75120 Uppsala, Sweden

Lei Tian - Department of Chemistry-Ångström Laboratory, Uppsala University, SE 75120 Uppsala, Sweden

Complete contact information is available at:

https://pubs.acs.org/10.1021/acsaem.1c00650

\section{Author Contributions}

The manuscript was written through contributions of all authors. All authors have given approval to the final version of the manuscript.

Notes

The authors declare no competing financial interest.

\section{ACKNOWLEDGMENTS}

We acknowledge the Wenner-Gren Foundation (UPD20180041) and the Wallenberg Academy Fellow for financial support. We also give our great thanks to Dr. Katerina Holá (UU), Stan J. Maassen (UT, BNT) for helpful discussion, Yawen Liu (UU) for the help with contact angle measurement, and the Molecular Biomimetics group and Polymer Chemistry group for sharing instruments.

\section{ABBREVIATIONS}

PFBT = poly[(9,9-di- $n$-octylfluorenyl-2,7-diyl)-alt-(benzo$[2,1,3]$ thiadiazol-4,8-diyl $)]$

THF $=$ tetrahydrofuran

SEM = scanning electron microscope

TCSPC $=$ time-correlated single photon counting

$\mathrm{GC}=$ gas chromatography

\section{REFERENCES}

(1) Arnold, W.; Clayton, R. K. The First Step in Photosynthesis: Evidence for Its Electronic Nature. Proc. Natl. Acad. Sci. U. S. A. 1960, 46 (6), 769-776.

(2) Mann, N. H.; Cook, A.; Millard, A.; Bailey, S.; Clokie, M. Bacterial Photosynthesis Genes in a Virus. Nature 2003, 424 (6950), 741.

(3) Zhang, T.; Lin, W. Metal-Organic Frameworks for Artificial Photosynthesis and Photocatalysis. Chem. Soc. Rev. 2014, 43 (16), $5982-5993$

(4) Fujishima, A.; Honda, K. Electrochemical Photolysis of Water at a Semiconductor Electrode. Nature 1972, 238 (5358), 37-38.

(5) Liu, R.; Huang, H.; Li, H.; Liu, Y.; Zhong, J.; Li, Y.; Zhang, S.; Kang, Z. Metal Nanoparticle/Carbon Quantum Dot Composite as a Photocatalyst for High-Efficiency Cyclohexane Oxidation. ACS Catal. 2014, 4 (1), 328-336.

(6) Wang, X.; Jiang, X.; Sharman, E.; Yang, L.; Li, X.; Zhang, G.; Zhao, J.; Luo, Y.; Jiang, J. Isolating Hydrogen from Oxygen in Photocatalytic Water Splitting with a Carbon-Quantum-Dot/CarbonNitride Hybrid. J. Mater. Chem. A 2019, 7 (11), 6143-6148.

(7) Bai, Y.; Wilbraham, L.; Slater, B. J.; Zwijnenburg, M. A.; Sprick, R. S.; Cooper, A. I. Accelerated Discovery of Organic Polymer Photocatalysts for Hydrogen Evolution from Water through the Integration of Experiment and Theory. J. Am. Chem. Soc. 2019, 141 (22), 9063-9071. 
(8) Chen, Y.; Ji, G.; Guo, S.; Yu, B.; Zhao, Y.; Wu, Y.; Zhang, H.; Liu, Z.; Han, B.; Liu, Z. Visible-Light-Driven Conversion of $\mathrm{CO} 2$ from Air to $\mathrm{CO}$ Using an Ionic Liquid and a Conjugated Polymer. Green Chem. 2017, 19 (24), 5777-5781.

(9) Fu, Z.; Vogel, A.; Zwijnenburg, M. A.; Cooper, A. I.; Sprick, R. S. Photocatalytic Syngas Production Using Conjugated Organic Polymers. J. Mater. Chem. A 2021, 9 (7), 4291-4296.

(10) Freeman, L. A.; Obi, A. D.; Machost, H. R.; Molino, A.; Nichols, A. W.; Dickie, D. A.; Wilson, D. J. D.; Machan, C. W.; Gilliard, R. J. Soluble, Crystalline, and Thermally Stable Alkali CO2and Carbonite (CO22-) Clusters Supported by Cyclic(Alkyl)(Amino) Carbenes. Chem. Sci. 2021, 12 (10), 3544-3550.

(11) Dai, C.; Liu, B. Conjugated Polymers for Visible-Light-Driven Photocatalysis. Energy Environ. Sci. 2020, 13 (1), 24-52.

(12) Liu, A.; Gedda, L.; Axelsson, M.; Pavliuk, M.; Edwards, K.; Hammarström, L.; Tian, H. Panchromatic Ternary Polymer Dots Involving Sub-Picosecond Energy and Charge Transfer for Efficient and Stable Photocatalytic Hydrogen Evolution. J. Am. Chem. Soc. 2021, 143 (7), 2875-2885.

(13) Wang, L.; Fernández-Terán, R.; Zhang, L.; Fernandes, D. L. A.; Tian, L.; Chen, H.; Tian, H. Organic Polymer Dots as Photocatalysts for Visible Light-Driven Hydrogen Generation. Angew. Chem., Int. Ed. 2016, 55 (40), 12306-12310.

(14) Elewa, A. M.; Elsayed, M. H.; El-Mahdy, A. F. M.; Chang, C.L.; Ting, L.-Y.; Lin, W.-C.; Lu, C.-Y.; Chou, H.-H. Triptycene-Based Discontinuously-Conjugated Covalent Organic Polymer Photocatalysts for Visible-Light-Driven Hydrogen Evolution from Water. Appl. Catal., B 2021, 285, 119802.

(15) Elsayed, M. H.; Jayakumar, J.; Abdellah, M.; Mansoure, T. H.; Zheng, K.; Elewa, A. M.; Chang, C.-L.; Ting, L.-Y.; Lin, W.-C.; Yu, H.; Wang, W.-H.; Chung, C.-C.; Chou, H.-H. Visible-Light-Driven Hydrogen Evolution Using Nitrogen-Doped Carbon Quantum DotImplanted Polymer Dots as Metal-Free Photocatalysts. Appl. Catal., B 2021, 283, 119659.

(16) Kosco, J.; Bidwell, M.; Cha, H.; Martin, T.; Howells, C. T.; Sachs, M.; Anjum, D. H.; Gonzalez Lopez, S.; Zou, L.; Wadsworth, A.; Zhang, W.; Zhang, L.; Tellam, J.; Sougrat, R.; Laquai, F.; DeLongchamp, D. M.; Durrant, J. R.; McCulloch, I. Enhanced Photocatalytic Hydrogen Evolution from Organic Semiconductor Heterojunction Nanoparticles. Nat. Mater. 2020, 19 (5), 559-565.

(17) Yang, H.; Li, X.; Sprick, R. S.; Cooper, A. I. Conjugated Polymer Donor-Molecular Acceptor Nanohybrids for Photocatalytic Hydrogen Evolution. Chem. Commun. 2020, 56 (50), 6790-6793.

(18) Aitchison, C. M.; Sprick, R. S.; Cooper, A. I. Emulsion Polymerization Derived Organic Photocatalysts for Improved LightDriven Hydrogen Evolution. J. Mater. Chem. A 2019, 7 (6), 24902496.

(19) Gupta, R.; Peveler, W. J.; Lix, K.; Algar, W. R. Comparison of Semiconducting Polymer Dots and Semiconductor Quantum Dots for Smartphone-Based Fluorescence Assays. Anal. Chem. 2019, 91 (17), 10955-10960.

(20) Sivula, K. Are Organic Semiconductors Viable for Robust, High-Efficiency Artificial Photosynthesis? ACS Energy Lett. 2020, 5 (6), 1970-1973.

(21) Sprick, R. S.; Little, M. A.; Cooper, A. I. Organic Heterojunctions for Direct Solar Fuel Generation. Commun. Chem. 2020, 3 (1), 40.

(22) Liu, A.; Tai, C.-W.; Holá, K.; Tian, H. Hollow Polymer Dots: Nature-Mimicking Architecture for Efficient Photocatalytic Hydrogen Evolution Reaction. J. Mater. Chem. A 2019, 7 (9), 4797-4803.

(23) Khan, M. A.; Armes, S. P. Synthesis and Characterization of Micrometer-Sized Poly(3,4-Ethylenedioxythiophene)-Coated Polystyrene Latexes. Langmuir 1999, 15 (10), 3469-3475.

(24) Sachs, M.; Cha, H.; Kosco, J.; Aitchison, C. M.; Francàs, L.; Corby, S.; Chiang, C.-L.; Wilson, A. A.; Godin, R.; Fahey-Williams, A.; Cooper, A. I.; Sprick, R. S.; McCulloch, I.; Durrant, J. R. Tracking Charge Transfer to Residual Metal Clusters in Conjugated Polymers for Photocatalytic Hydrogen Evolution. J. Am. Chem. Soc. 2020, 142 (34), 14574-14587.
(25) Pati, P. B.; Damas, G.; Tian, L.; Fernandes, D. L. A.; Zhang, L.; Pehlivan, I. B.; Edvinsson, T.; Araujo, C. M.; Tian, H. An Experimental and Theoretical Study of an Efficient Polymer NanoPhotocatalyst for Hydrogen Evolution. Energy Environ. Sci. 2017, 10 (6), 1372-1376.

(26) Aubry, J.; Ganachaud, F.; Cohen Addad, J.-P.; Cabane, B. Nanoprecipitation of Polymethylmethacrylate by Solvent Shifting:1. Boundaries. Langmuir 2009, 25 (4), 1970-1979.

(27) Abstiens, K.; Goepferich, A. M. Microfluidic Manufacturing Improves Polydispersity of Multicomponent Polymeric Nanoparticles. J. Drug Delivery Sci. Technol. 2019, 49, 433-439.

(28) MacFarlane, L. R.; Shaikh, H.; Garcia-Hernandez, J. D.; Vespa, M.; Fukui, T.; Manners, I. Functional Nanoparticles through $\pi$ Conjugated Polymer Self-Assembly. Nat. Rev. Mater. 2021, 6 (1), 726.

(29) Baldan, A. Review Progress in Ostwald Ripening Theories and Their Applications to Nickel-Base Superalloys Part I: Ostwald Ripening Theories. J. Mater. Sci. 2002, 37 (11), 2171-2202.

(30) Brinker, C. J.; Lu, Y.; Sellinger, A.; Fan, H. EvaporationInduced Self-Assembly: Nanostructures Made Easy. Adv. Mater. 1999, 11 (7), 579-585.

(31) Kelly, T. L.; Yano, K.; Wolf, M. O. Supercapacitive Properties of PEDOT and Carbon Colloidal Microspheres. ACS Appl. Mater. Interfaces 2009, 1 (11), 2536-2543. 